TecnoLógicas

ISSN-p 0123-7799

ISSN-e 2256-5337

Vol. 20, No. 40, pp. 71-81

Sep-dic de 2017

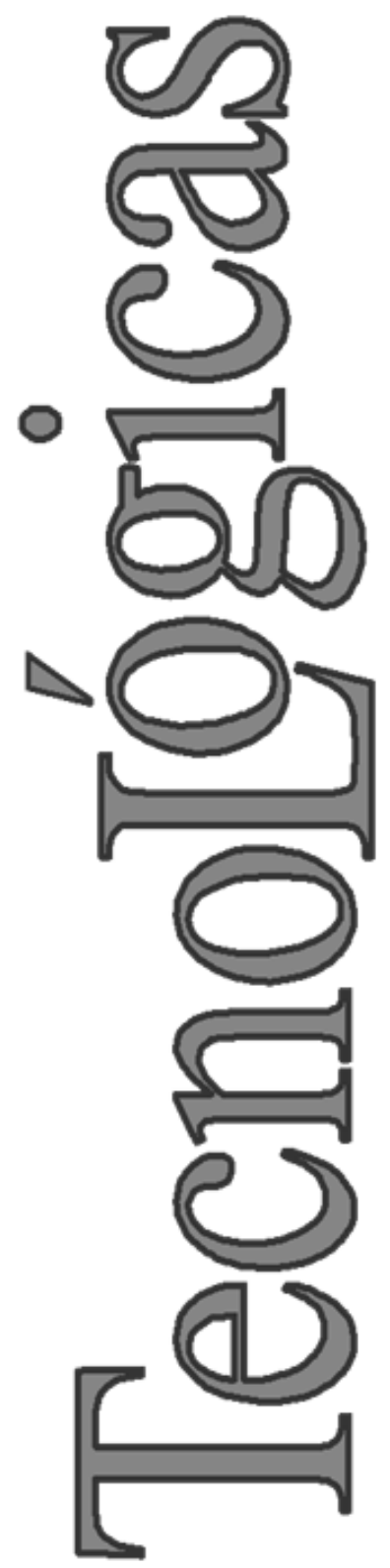

(C) Copyright 2015 por autores y Tecno Lógicas Este trabajo está licenciado bajo una Licencia Internacional Creative Commons Atribución (CC BY)

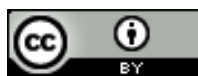

Artículo de Investigación/Research Article

\section{Influencia de la altura del lecho sobre el comportamiento dinámico de columna de lecho fijo en la biosorción de mercurio}

\section{Influence of the bed height on the dynamic behavior of a fixed-bed column during mercury biosorption}

\author{
Candelaria N. Tejada-Tovar ${ }^{1}$, Angel Villabona-Ortíz ${ }^{2}$, \\ Gemaly Alvarez-Bajaire ${ }^{3}$, Leidy Jattin-Torres ${ }^{4}$ y \\ Clemente Granados-Conde ${ }^{5}$
}

Recibido: 12 de junio de 2017

Aceptado: 30 de agosto de 2017

\section{Cómo citar / How to cite}

C. N. Tejada-Tovar, A. Villabona-Ortíz, G. Alvarez-Bajaire, L. JattinTorres y C. Granados-Conde, Influencia de la altura del lecho sobre el comportamiento dinámico de columna de lecho fijo en la biosorción de mercurio. TecnoLógicas, vol. 20, no. 40, pp. 71-81, 2017.

1 M.Sc. en Ingeniería Ambiental. M.Sc. Educación, Grupo de Investigación en Diseño de procesos y Aprovechamiento de Biomasas IDAB, Facultad de Ingeniería, Universidad de Cartagena, Cartagena-Colombia, ctejadat@unicartagena.edu.co

2 M.Sc. en Ingeniería Ambiental, Grupo de Investigación en Diseño de procesos y Aprovechamiento de Biomasas IDAB, Facultad de Ingeniería, Universidad de Cartagena, Cartagena-Colombia, avillabonao@unicartagena.edu.co

3 Ingeniera Química, Grupo de Investigación en Diseño de procesos y Aprovechamiento de Biomasas IDAB, Facultad de Ingeniería, Universidad de Cartagena, Cartagena-Colombia, gemalita92@hotmail.com

4 Ingeniera Química, Grupo de Investigación en Diseño de procesos y Aprovechamiento de Biomasas IDAB, Facultad de Ingeniería, Universidad de Cartagena, Cartagena-Colombia,jattincita_3004@hotmail.com

5 M.Sc. en Ciencia y Tecnología de los Alimentos, Grupo de investigación Ingeniería, Innovación, Calidad Alimentaria y Salud -INCAS, Facultad de Ingeniería, Universidad de Cartagena, Cartagena-Colombia, cgranadosc@unicartagena.edu.co 



\section{Resumen}

Se determinó el impacto de la altura del lecho en la remoción de $\mathrm{Hg}$ (II), presente en solución sobre biomasa residual de cáscara de cacao en sistema continuo de lecho fijo, evaluando el rendimiento de la densidad de empaque. El trabajo experimental estuvo fundamentado en: preparación de la biomasa, diseño y montaje de la unidad de adsorción y modelado matemático, considerando como variables intervinientes en el proceso la concentración inicial del metal, $\mathrm{pH}$, velocidad de flujo y tamaño de partícula y como variable independiente incidente la densidad de empaque $\mathrm{mg}$ biomasa/volumen del lecho, traducido en altura en cm de empaque. Los análisis FTIR de la cáscara muestran la presencia de grupos funcionales que favorecen la adsorción de iones metálicos. La concentración de $\mathrm{Hg}$ (II) se midió por espectroscopia UV/vis; se obtuvo una capacidad máxima de adsorción fue de $99.62 \%$ para el lecho de $10 \mathrm{~g}(7.5 \mathrm{~cm})$. Además, el modelo de Thomas fue el que mejor ajustó los datos experimentales. A partir de estos resultados, se concluye que la cáscara de cacao tiene potencial para ser utilizado como bioadsorbente de $\mathrm{Hg}$ (II) en solución y que el aumento de la altura del lecho en el sistema continuo favoreció la remoción del contaminante.

\section{Palabras clave}

Bioadsorbente, cáscara de cacao, sistema continuo, adsorción molecular, modelado matemático.

\section{Abstract}

This study evaluated the packing density of residual biomass of cocoa bean husk as adsorbent of $\mathrm{Hg}$ (II) dissolved in an aqueous solution inside a continuous fixed-bed system. The effects of the height of the bed on the removal of the pollutant were evaluated. This experimental work was based on biomass preparation, adsorber design and assembly, and mathematical modelling. The variables considered in the process were initial concentration of the metal, $\mathrm{pH}$, flow rate and particle size. The incident-independent variable was the packing density ( $\mathrm{mg}$ biomass/bed volume), which translated into the height $(\mathrm{cm})$ of the packing. The FTIR analyses of the husk revealed the presence of functional groups in the spectrum that favour the adsorption of the metal. The residual concentration of the solution was measured by UV/Vis spectroscopy; the maximum adsorption capacity was $99.62 \%$, by the $10 \mathrm{~g}(7.5 \mathrm{~cm})$ bed. In addition, the Thomas model was the best-fitting for the experimental data. On the basis of these results, we concluded that cocoa bean husk has potential to be used as bioadsorbent of $\mathrm{Hg}$ (II) from aqueous solutions and that the increase in bed height in the continuous system favours the removal of the pollutant.

\section{Keywords}

Bioadsorbent, cocoa shell, continuous system, molecular adsorption, mathematical modelling. 


\section{INTRODUCCIÓN}

Uno de los productos agroalimentarios de mayor inserción en el mercado internacional es el cacao (Theobroma cacao L.), lo cual ha generado que su procesamiento produzca grandes volúmenes de desechos [1]. Esto debido a que, durante la explotación cacaotera sólo se usufructúa la semilla, que se traduce en el $10 \%$ de la masa total del producto. Los subproductos generados, cáscara y la pulpa, al ser depositados en los suelos se consideran un foco para la propagación de Moniliophthora roreri y Phytophthora spp., los cuales ocasionan mermas mercantiles, relacionadas con la descomposición de la mazorca originada por estos hongos [2]. De igual forma, en Colombia las enfermedades moniliasis y pudrición negra del fruto, han sido reportadas en plantíos cacaoteros; los resultados de esta investigación ayudaron a la comprensión del ciclo espacial en la Orinoquía Colombiana de estas enfermedades en tres sembradíos, ligados a la inadecuada disposición final de estos restos [3].

Los vertimientos de aguas contaminadas con metales pesados a los cuerpos de agua superficiales, provenientes de actividades antrópicas e industriales como metal, textiles, curtiembres, minería, papel, refinerías de petróleo, y manufacturas químicas; representa una amenaza para la salud pública y ambiente, debido a la toxicidad por bioacumulación en los organismos de sales hidrosolubles de iones metálicos como el mercurio $(\mathrm{Hg})$, contaminando así las cadenas tróficas, suscitando padecimientos como: ceguera, amnesia, raquitismo, miastenia, cáncer, insuficiencia renal, incluso la muerte [4] [5] [6].

Diferentes tecnologías se han usado para la eliminación de metales presentes en efluentes de origen industrial, entre las que se cuentan: la precipitación, ultrafiltración, ósmosis inversa, intercambio iónico, extracción por membranas y solventes, electrodiálisis, entre otras [7] [8] [9]. Estos métodos pueden remover hasta el $99 \%$ de estos metales, sin embargo, su alto costo de instalación y mantenimiento, la generación de lodos y su poca eficiencia a bajas concentraciones dificulta su aplicación a las pequeñas y medianas empresas [10]. Por lo tanto, se han utilizado procesos sostenibles para este fin, empleando residuos agrícolas como bioadsorbentes, los cuales son eficientes, económicos, abundantes $\mathrm{y}$ reutilizables [11].

Adicionalmente, en la remoción de iones en sistema batch y continuo, se han usado como adsorbente carbones activados a partir de agujas de pino, cáscara de Swietenia mahagoni, nanotubos de carbono de paredes múltiples con grupos amino y tiolados, residuos de cacao, bagazo de palma, cáscaras de ñame, entre otros, demostrando el potencial de las biomasas lignocelulósicas en la remediación de aguas [4] [1215]. En contexto, el presente trabajo evaluó el desempeño de la densidad de empaque de biomasa residual de cáscara de cacao como adsorbente de $\mathrm{Hg}$ (II), disuelto en solución acuosa, en un sistema continuo de lecho fijo determinando el efecto de la altura del lecho en la remoción de este contaminante.

\section{MATERIALES Y MÉTODOS}

\subsection{Preparación del bioadsorbente}

La cáscara de cacao fue recolectada de cultivos cacaoteros del departamento de Bolívar en Colombia. Este residuo se lavó con agua destilada para remover las impurezas hasta que el agua no presentó coloración alguna. Terminada esta etapa, se secó el material en el horno a $90^{\circ} \mathrm{C}$ durante $24 \mathrm{~h}$ con el fin de retirar todo el exceso de agua del residuo. El material seco se llevó a molienda y se tamizó para obtener el tamaño de partícula seleccionado $(0.5 \mathrm{~mm})$ [1]. 


\subsection{Caracterización del bioadsorbente}

Se realizó el análisis fisicoquímico a la biomasa por el método EAA (Espectroscopia de Absorción Atómica) para determinar los elementos químicos: calcio, sodio, potasio, hierro, cobre y magnesio. Y un análisis elemental para determinar el porcentaje de carbono (método AOAC 949.14), hidrogeno (método AOAC 949.14), porcentaje de nitrógeno (método AOAC 984.13), contenido de azufre (digestión), porcentaje de cenizas y pectina (termogravimetría y digestión acida), porcentaje de lignina, celulosa y hemicelulosa (Fotocolorimetria y digestión). Análisis FTIR a la biomasa antes y después de la remoción, para identificar los grupos funcionales intervinientes en la adsorción [9].

\subsection{Preparación del agua residual sintética}

Para preparar el agua residual sintética se usó $0,135 \mathrm{~g}$ de cloruro de mercurio (II) $\left(\mathrm{HgCl}_{2}\right)$ disuelto en $1 \mathrm{~L}$ de agua desionizada obteniendo la concentración deseada de
100 ppm y se ajustó el pH a 6 usando $\mathrm{NaOH}[4]$.

\subsection{Comportamiento dinámico de la colum- na de adsorción}

Las pruebas de adsorción se realizaron en un equipo piloto que consta de cuatro columnas, con diámetro de $3.5 \mathrm{~cm}$ y altura de $18.5 \mathrm{~cm}$, equipado con un tanque de alimentación con el agua sintética preparada, una bomba sumergible que alimenta el agua hacia el tanque de distribución donde se mantiene a un nivel constante por rebose, después por gravedad se distribuye el agua a las columnas, como se muestra en la Fig. 1, con control de flujo manual. Se realizó un montaje experimental variando la altura del lecho durante 7.5 h, se utilizó para este estudio un lecho empacado de $4 \mathrm{y}$ $7.5 \mathrm{~cm}$, por el cual pasó la solución de agua sintética preparada a una velocidad de flujo de $6 \mathrm{~mL} / \mathrm{min}$ [15]. Los ensayos se realizaron con réplica para la confiabilidad de los resultados obtenidos.

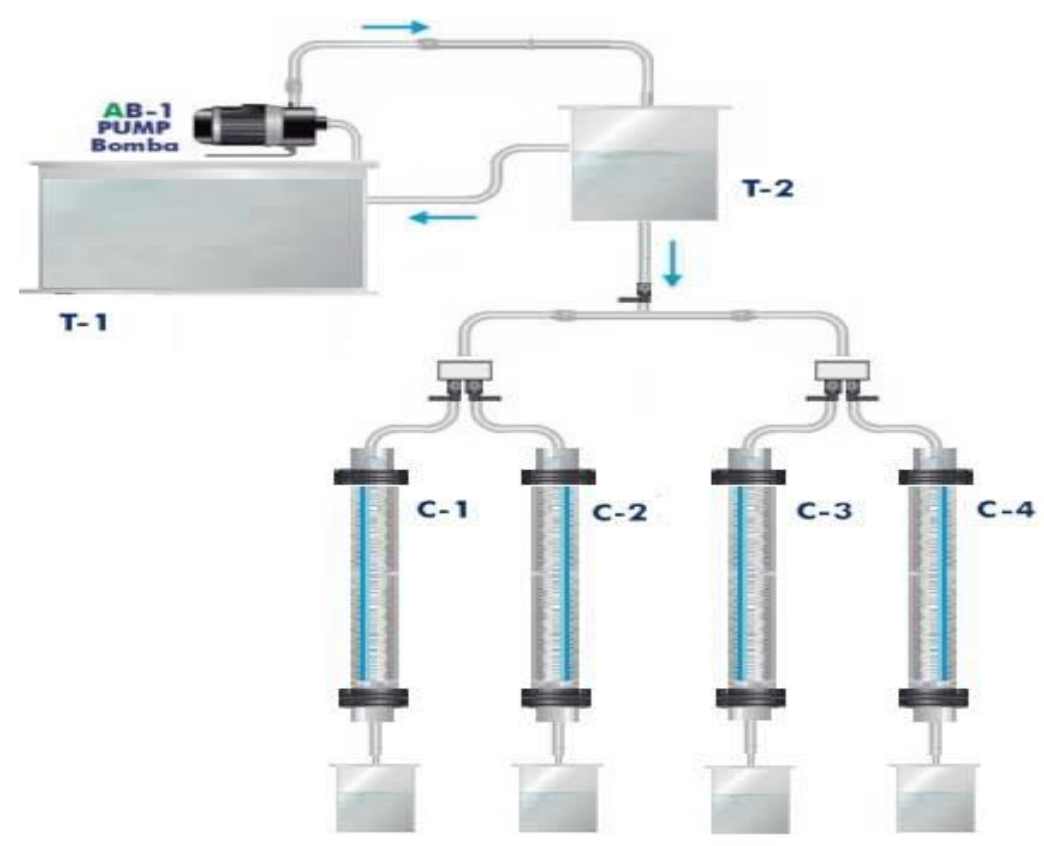

Fig. 1. Modelo experimental empleado [15]. 


\section{RESULTADOS Y DISCUSIÓN}

\subsection{Caracterización: análisis proximal ele- mental y FIIR}

En los resultados presentados en la Tabla 1 , se observa un alto contenido de carbono en el adsorbente (50.35\%); y que los contenidos de celulosa, lignina, pectina $\mathrm{y}$ hemicelulosa tienen un porcentaje de 19.82 $\%, 12.66 \%, 9.54 \%$ y $9.45 \%$ respectivamente.

Los espectros FTIR de la cáscara de cacao se localizaron entre 4000 y $400 \mathrm{~cm}^{-1}$. La Fig. 2 representa el cambio del espectro de la biomasa tratada solo con agua destilada, con respecto al obtenido después de colocar la biomasa en contacto con la solución de mercurio.

En la Tabla 2 se muestra el estado de energía vibracional y el grupo funcional correspondiente a la longitud de onda del espectro, permitiendo relacionar estas tipologías con los diferentes componentes y establecer cualitativamente las especies que pueden propiciar la adsorción; y en la Tabla 3 los grupos funcionales que se mantuvieron luego de ser utilizada la biomasa como adsorbente.

Tabla 1. Análisis fisicoquímico de la biomasa de cacao. Fuente: autores.

\begin{tabular}{lcl}
\hline Parámetros & Valor & Método Analítico \\
\hline Carbono, \% & 50.4 & AOAC 949.14 \\
\hline Hidrogeno, \% & 5.1 & AOAC 949.14 \\
\hline Nitrógeno, \% & 1.3 & AOAC 984.13 \\
\hline Azufre, ppm & 0.6 & Digestion-Nefelometrica \\
\hline Cenizas, $\%$ & 7.8 & Termogravimetria \\
\hline Pectina, \% & 9.5 & Digestión acida-Termogravimetria \\
\hline Lignina, \% & 12.7 & Fotocolorimetria \\
\hline Celulosa, \% & 19.8 & Digestión-Termogravimetria \\
\hline Hemicelulosa, \% & 9.5 & Digestión-Termogravimetria
\end{tabular}

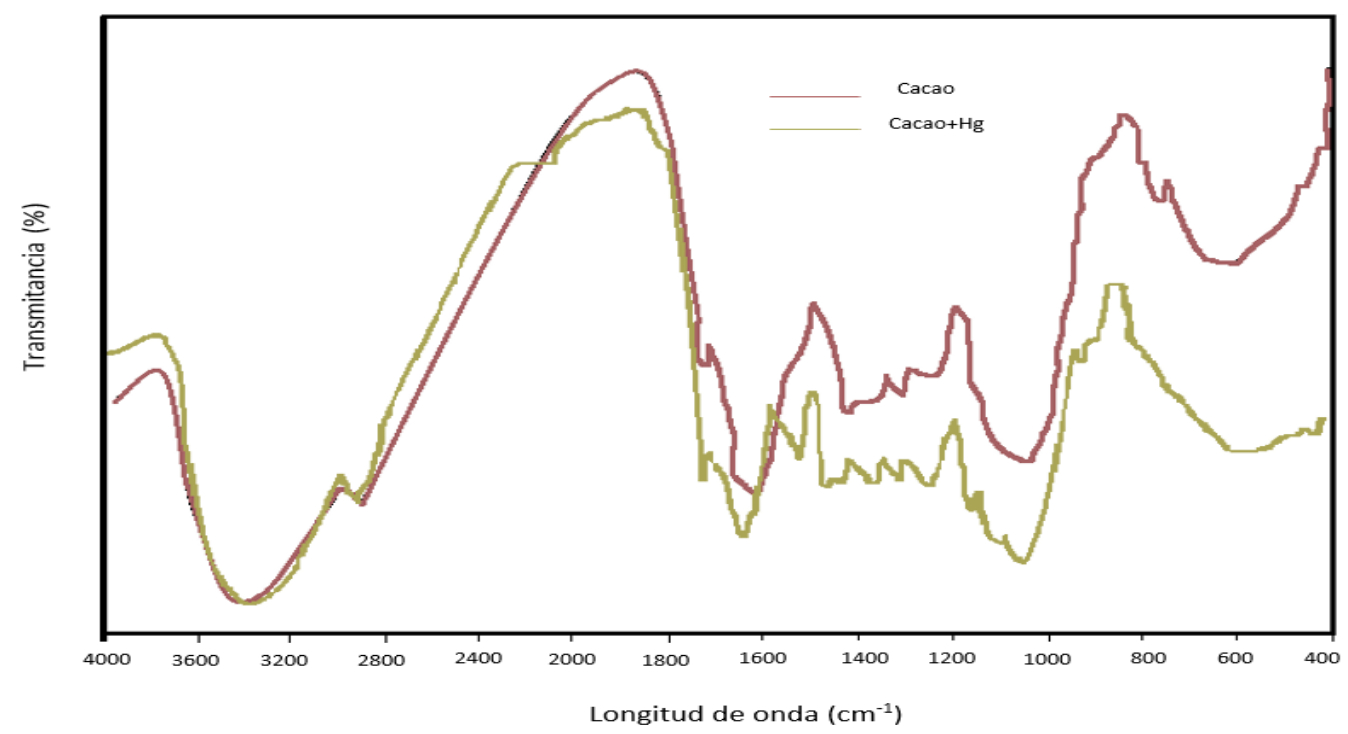

Fig. 2. Espectro infrarrojo para la biomasa de cacao. Fuente: autores. 
Influencia de la altura del lecho sobre el comportamiento dinámico de columna de lecho fijo en la biosorción de mercurio

Tabla 2. Grupos funcionales en la cáscara de cacao. Fuente: autores.

\begin{tabular}{|c|c|c|}
\hline Longitud de Onda $\left(\mathrm{cm}^{-1}\right)$ & Tipo de Vibración & Grupo Funcional \\
\hline $422.43,439.79,457.15$ & Si-O extensión & Grupo funcional sílica \\
\hline $668.36-518.87$ & C-X extensión & Organo-halógenos \\
\hline $780.24,817.85$ & $\mathrm{C}=\mathrm{C}-\mathrm{H}$ deformación & Alifáticos insaturados \\
\hline 896.94 & C-H flexión & Aromático \\
\hline $1155.41-1035.82$ & $\mathrm{C}=\mathrm{S}$ extensión & Tioésteres, tioureas, tioamidas \\
\hline $1260.54,1284.65,1318.40$ & C-O-C extensión & Éteres (aromático, olefínicos o alifáticos) \\
\hline $1372.41,1453.43$ & H-C-H flexión & Metilo, metileno \\
\hline $1543.12-1506.47$ & -NOz estiramiento asimétrico & Nitro- compuestos orgánicos \\
\hline $1873.93-1558.55$ & $\mathrm{C}=\mathrm{O}$ extensión & $\begin{array}{l}\text { Ésteres, cetonas, amidas, ácidos carboxílicos y sus } \\
\text { sales, anhídridos de ácido }\end{array}$ \\
\hline 1998.34-1926.97 & $\mathrm{C}=\mathrm{C}$ & Hidrocarburos aromáticos \\
\hline $2026.31,2165.19,2196.05$ & $\mathrm{C} \equiv \mathrm{C}$ & $\begin{array}{l}\text { Hidrocarburos alifáticos: los alquinos, alenos, cianato, } \\
\text { isocianato, nitrilo, isocianuros, azidas de diazonio, } \\
\text { sales, cetonas, tiocianatos, isotiocianatos }\end{array}$ \\
\hline $2374.47-2216.30$ & $\mathrm{C} \equiv \mathrm{N}$ & Grupo funcional nitrógeno \\
\hline 2928.07, 2993.65 & C-H extensión & $\begin{array}{l}\text { Hidrocarburos alifáticos: metilo, metileno, grupos } \\
\text { metino }\end{array}$ \\
\hline $3167.25,3187.51$ & C-H extensión & Insaturados: compuestos aromáticos y olefínicos \\
\hline $3952.31-3265.63$ & $\mathrm{OH}^{-}$ & Grupo hidroxilo \\
\hline
\end{tabular}

Tabla 3. Especies orgánicas funcionales presentes en la cáscara de cacao después de remover mercurio. Fuente: autores.

\begin{tabular}{ccl}
\hline $\begin{array}{c}\text { No Longitud de Onda } \\
\text { IR Frecuencia }\left(\mathbf{c m}^{-1}\right)\end{array}$ & Tipo de Vibración & Grupo Funcional \\
\hline $557.45,655.83$ & C-X extensión & Organohalógenos \\
\hline 895.97 & $\mathrm{C}-\mathrm{H}$ flexión & Aromático \\
\hline $1156.37-1032.93$ & $\mathrm{C}=$ S extensión & Tioésteres, tioureas, tioamidas \\
\hline $1258.61,1318.40$ & $\mathrm{C}-\mathrm{O}-\mathrm{C}$ extensión & Éteres (aromático, olefínicos o alifáticos) \\
\hline 1622.20 & $\mathrm{C}=\mathrm{O}$ extensión & $\begin{array}{l}\text { Ésteres, cetonas, amidas, ácidos carboxílicos y sus } \\
\text { sales, anhídridos de ácido }\end{array}$ \\
\hline 2927.10 & $\mathrm{C}-\mathrm{H}$ extensión & $\begin{array}{l}\text { Hidrocarburos Alifáticos: Metilo, metileno, grupos } \\
\text { metino }\end{array}$ \\
\hline $3561.71-3338.92$ & $\mathrm{OH}-$ & Grupo hidroxilo \\
\hline
\end{tabular}

\subsection{Efecto de la altura del lecho empacado}

Se valoró estableciendo el porcentaje de adsorción y el tiempo de ruptura de cada columna para la remoción de mercurio. De acuerdo con la Tabla 4, el porcentaje de remoción aumenta con la altura del lecho empacado de biomasa, siendo la altura de $7.5 \mathrm{~cm}$ la que favorece el proceso respecto a la de $4 \mathrm{~cm}$.

En este estudio el tiempo de ruptura $\left(\mathrm{T}_{\text {rup }}\right)$ se establece como el lapso de tiempo que transcurre hasta que la concentración del adsorbato alcance $5 \%$ de la concentración inicial. El Qrup, establecida según (1), se define como cantidad de contaminante adsorbido (mg) por masa (g) adsorbente en el tiempo de ruptura:

$$
Q_{\text {rup }}\left[\frac{\boldsymbol{m g}}{\boldsymbol{g}}\right]=\frac{Q_{v} t_{5 \%} C_{o}}{m}
$$

Donde, $t_{(5 \%)}$ es el tiempo de servicio en minutos conseguido cuando la concentración de la solución en la salida es el $5 \%$ de la concentración de entrada, $\mathrm{C}_{0}$ es la concentración en $\mathrm{mg} / \mathrm{L}$ de metal en la entrada a la columna, $\mathrm{Q}_{\mathrm{v}}$ es el caudal ( $\left.\mathrm{L} / \mathrm{min}\right)$ y $\mathrm{m}$ es la cantidad de biomasa de cascara de cacao. Los parámetros generados del análisis de los datos de las curvas de ruptura se muestran en la Tabla 5: 
Influencia de la altura del lecho sobre el comportamiento dinámico de columna de lecho fijo en la biosorción de mercurio

Tabla 4. Porcentaje de remoción de cada columna para la adsorción de Hg (II). Fuente: autores.

\begin{tabular}{ccccc}
\hline \multirow{2}{*}{$\begin{array}{c}\text { Tiempo } \\
(\text { min) }\end{array}$} & \multicolumn{2}{c}{ Lecho 5 g } & \multicolumn{2}{c}{ Lecho 10 g } \\
\cline { 2 - 5 } & Remoción (mg/L) & \% Adsorción & Remoción (mg/L) & \% Adsorción \\
\hline 10 & 12,39 & 87,61 & 0,37 & 99,63 \\
\hline 30 & 16,49 & 83,50 & 0,426 & 99,58 \\
\hline 60 & 17,28 & 82,72 & 1,00 & 99,00 \\
\hline 90 & 17,56 & 82,44 & 3,11 & 96,89 \\
\hline 120 & 18,96 & 81,04 & 94,91 \\
\hline 150 & 19,23 & 80,77 & 92,95 \\
\hline 180 & 19,61 & 80,39 & 7,05 & 90,94 \\
\hline 210 & 20,70 & 79,30 & 9,06 & 88,83 \\
\hline 240 & 21,48 & 78,52 & 11,17 & 88,19 \\
\hline 270 & 22,53 & 77,47 & 11,82 & 86,53 \\
\hline 300 & 23,59 & 76,41 & 83,42 \\
\hline 330 & 24,11 & 76,47 & 80,28 \\
\hline 360 & 25,35 & 74,65 & 19,72 & 78,35 \\
\hline 390 & 27,33 & 21,65 & 75,67 \\
\hline 420 & 30,25 & 24,63 & 70,59 \\
\hline 450 & 32,02 & 69,75 & 29,41 & 67,61 \\
\hline
\end{tabular}

Tabla 5. Parámetros establecidos de la curva de ruptura de remoción de Hg (II) sobre cáscara de cacao. Fuente: autores.

\begin{tabular}{lccccc}
\hline Metal & H (cm) & M (g) & $\mathbf{T}_{\text {rup }}(\mathbf{m i n})$ & $\mathbf{Q}_{\text {rup }}(\mathbf{m g} / \mathbf{g})$ & \% Remoción $\mathbf{n}_{\text {máx. }}$ \\
\hline \multirow{2}{*}{ Mercurio } & 4 & 5 & 0 & 0 & 87,61 \\
\hline & 7,5 & 10 & 120 & 7,2 & 99,62 \\
\hline
\end{tabular}

\subsection{Modelado de las curvas de ruptura}

La eficiencia de una columna de lecho empacado se establece mediante el concepto de curva de ruptura, la cual es una característica que permite determinar el tiempo de servicio y establecer la respuesta dinámica de la columna; el desempeño del ion retenido en lecho de cacao; se expresa en términos de la variación de la concentración inicial con respecto al tiempo, $\mathrm{C}_{t} / \mathrm{C}_{\mathrm{o}}$, la cual es una función del volumen del efluente a una altura dada, generando de esta manera la curva respectiva [15-19]. Con el fin de analizar el rendimiento de la columna se realizó el ajuste del modelo cinético de los datos experimentales a los siguientes modelos:

\subsubsection{Modelo de Adams-Bohart (AB)}

Este modelo es usado en la descripción de la parte inicial de la curva de ruptura, asumiendo que la velocidad de adsorción es proporcional a la capacidad de adsorción del adsorbente y a la concentración del soluto, y se enuncia en (2) [15, 17].

$$
\frac{C_{t}}{C_{0}}=\exp \left(\mathrm{k}_{\mathrm{AB}} \mathrm{C}_{0} \mathrm{t}-\mathrm{k}_{\mathrm{AB}} \mathrm{N}_{0} \frac{\mathrm{Z}}{\mathrm{F}}\right)
$$

En esta expresión, $\mathrm{k}_{\mathrm{AB}}$ es la constante cinética de adsorción $\left(\mathrm{L} \mathrm{mg}^{-1} \mathrm{~min}^{-1}\right), \mathrm{F}$ es la velocidad lineal $\left(\mathrm{cm} \min ^{-1}\right), \mathrm{Z}$ es la altura del lecho empacado y $\mathrm{N}_{0}$ es la concentración de saturación del adsorbente (mg L $\mathrm{L}^{-1}$ ); estos parámetros se establecen a partir del gráfico $\mathrm{C}_{t} / \mathrm{C}_{0} \mathrm{vs} \mathrm{t}$. 


\subsubsection{Modelo de Thomas (Th)}

Es utilizado para graficar y analizar los datos experimentales derivados de ensayos en columnas, con el fin de determinar la máxima capacidad de adsorción del material y su constante cinética; y se describe mediante (3) $[15,18]$ :

$$
\frac{C_{t}}{C_{0}}=\frac{1}{1+\exp \left(\mathrm{k}_{\mathrm{Th}} \mathrm{q}_{0} \mathrm{x} / \mathrm{v}-\mathrm{k}_{\mathrm{Th}} \mathrm{C}_{0} \mathrm{t}\right)}
$$

Donde, $\mathrm{k}_{\mathrm{Th}}$ es la constante cinética $(\mathrm{mL}$ $\left.\min ^{-1} \mathrm{mg}^{-1}\right)$, qo es la cantidad de soluto adsorbida por gramo de adsorbente (mg g$\left.{ }^{1}\right)$, x es la masa de adsorbente en la columna $(\mathrm{g}), \mathrm{C}_{0}$ es la concentración inicial del efluente $\left(\mathrm{mg} \mathrm{L}^{-1}\right), \mathrm{C}_{t}$ es la variación de la concentración del efluente en el tiempo $t$ $\left(\mathrm{mg} \mathrm{L}^{-1}\right)$ y $\mathrm{v}$ es el caudal de entrada $(\mathrm{mL}$ $\left.\min ^{-1}\right)$. El coeficiente cinético $\mathrm{k}_{\mathrm{Th}}$ y la capacidad de adsorción de la columna, qo, pueden ser determinados por regresión nolineal del gráfico $\mathrm{C}_{t} / \mathrm{C}_{\mathrm{o}}$ vs $\mathrm{t}$, a una velocidad de entrada dada.

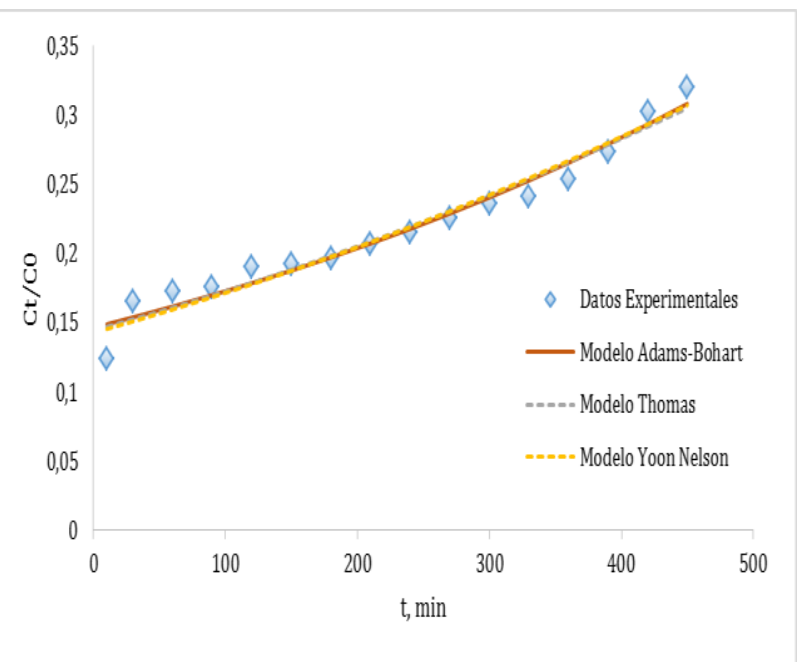

(a)

\subsubsection{Modelo de Yoon-Nelson (YN)}

El modelo linealizado para un sistema monocomponente se expresa en (4); y asume que la tasa de disminución en la probabilidad de adsorción para cada molécula de adsorbato es proporcional a la probabilidad de adsorción de adsorbato y la probabilidad de ruptura de adsorbato en el adsorbente. No requiere de datos detallados sobre las características del adsorbato, el tipo de adsorbente, y las propiedades físicas del lecho de adsorción [15, 17]:

$$
\frac{C_{t}}{C_{0}-C_{t}}=\exp \left(k_{Y N} t-\tau k_{Y N}\right)
$$

Donde kyn es la constante de velocidad $\left(\min ^{-1}\right)$ y $\tau(\min )$ el tiempo necesario para una penetración del adsorbato del $50 \%$.

En la Fig. 3(a) se muestra el comportamiento de la curva de ruptura de la adsorción de $\mathrm{Hg}$ (II) sobre una columna de $4 \mathrm{~cm}$ de cacao y el modelado de los datos experimentales para un tiempo de $450 \mathrm{~min}$. Por su parte, en la Fig. 3 (b), se observa el desempeño de la curva de rup tura de la columna de $7.5 \mathrm{~cm}$ para el mismo tiempo de operación y adsorbato.

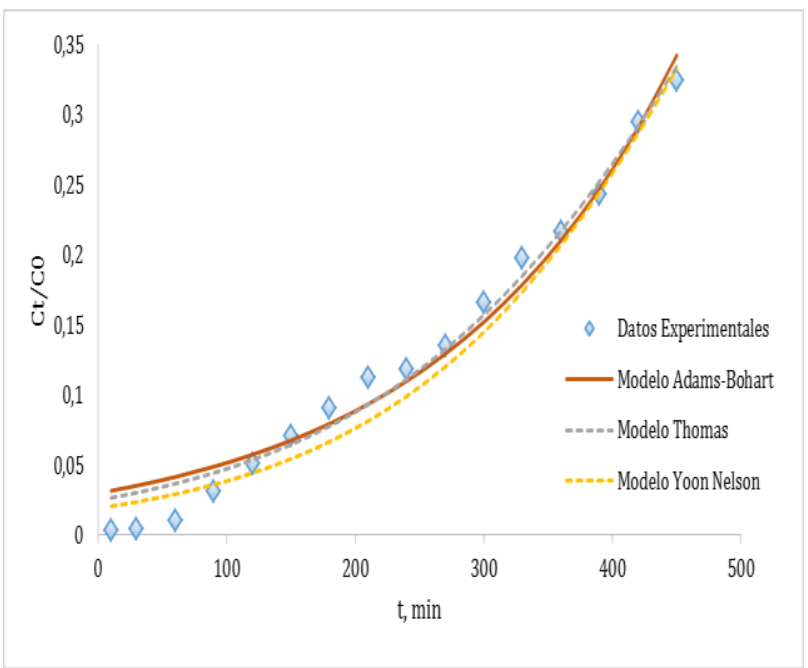

(b)

Fig. 3 (a). Ajuste de los modelos cinéticos de adsorción de $\mathrm{Hg}$ (II) de la columna empacada con de $4 \mathrm{~cm}$ (a) y $7.5 \mathrm{~cm}$ (b)

Fuente: autores. 
Influencia de la altura del lecho sobre el comportamiento dinámico de columna de lecho fijo en la biosorción de mercurio

Tabla 6. Parámetros ajuste de los modelos cinéticos de adsorción de mercurio sobre cacao en sistema continuo.

Fuente: autores.

\begin{tabular}{|c|c|c|c|}
\hline \multirow{2}{*}{ MODELO } & \multirow{2}{*}{ PARÁMETROS } & \multicolumn{2}{|c|}{$\mathrm{Hg}$} \\
\hline & & $\mathrm{H}=4 \mathrm{~cm}$ & $\mathrm{H}=7,5 \mathrm{~cm}$ \\
\hline \multirow{3}{*}{ Adams-Bohart } & $\mathrm{K}_{\mathrm{AB}}$ (L/mg.min) & 0 & $1 \times 10^{-4}$ \\
\hline & $\mathrm{N}_{0}(\mathrm{mg} / \mathrm{L})$ & 5087,99 & 1515,85 \\
\hline & $\mathrm{R}^{2}$ & 0,97 & 0,98 \\
\hline \multirow{3}{*}{ Thomas } & $\mathrm{K}_{\mathrm{TH}}(\mathrm{mL} / \mathrm{mg} \cdot \mathrm{min})$ & 0,02 & 0,06 \\
\hline & $\mathrm{q}_{0}(\mathrm{mg} / \mathrm{g})$ & 100,49 & 33,25 \\
\hline & $\mathrm{R}^{2}$ & 0,98 & 0,99 \\
\hline \multirow{3}{*}{ Yoon-Nelson } & $\mathrm{K}_{\mathrm{YN}}(\mathrm{mL} / \mathrm{mg} \cdot \mathrm{min})$ & $2,2 \times 10^{-3}$ & $7,2 \times 10^{3}$ \\
\hline & $\mathrm{t}(\mathrm{min})$ & 823,78 & 546,773 \\
\hline & $\mathrm{R}^{2}$ & 0,98 & 0,99 \\
\hline
\end{tabular}

En la Tabla 6 se observa que para la altura de lecho de 4 y $7.5 \mathrm{~cm}$, el modelo de Thomas fue el que mejor ajustó los datos experimentales, con un valor de correlación de $\mathrm{R}^{2}=0.98$ y 0.99 , respectivamente.

\section{DISCUSIÓN}

El análisis químico proximal de la Tabla 1 presenta los principales componentes propiciadores de la remoción de iones metálicos, puesto que en su estructura cuentan con gran presencia grupos $\mathrm{OH}$ (hidroxilos y fenólicos); por lo tanto es de esperar que la cáscara de cacao sea un adsorbente con gran capacidad de remoción, ya que su naturaleza lignocelulósica confirma la presencia de compuestos con largas cadenas poliméricas (celulosa, hemicelulosa, pectina y lignina), que son reconocidos por la gran cantidad de grupos hidroxilos y carboxilos que presentan en su disposición [4-6], [15], [16]. En el análisis del espectro FTIR de la Fig. 2, se identificó la presencia de: hidrocarburos alifáticos, aromáticos e insaturados con una energía vibracional variable, compuestos orgánicos, halógenos, metilo, sílica y nitrógeno y grupos hidroxilo, de acuerdo con la Tabla 2. La vibración en la región entre 3900 a $3200 \mathrm{~cm}^{-1}$ confirma la presencia de grupos $\mathrm{OH}$, los cuales benefician la remoción de metales pesados, debido a que los grupos de carga aniónica en la superficie del sorbente atraen electrostáticamente a los cationes. El espectro presenta una tendencia decreciente en este rango, porque el enlace de hidrógeno tiende a ampliar los picos y desplazarlos hacia números de onda más bajos. Por tanto, la forma ancha y de gran intensidad del pico sobre 3627.29- $3167.25 \mathrm{~cm}^{-1}$ es típica [9]. Con base en lo reportado en la Tabla 3, se puede decir que el espectro de la biomasa luego de la adsorción de mercurio y el espectro de la biomasa natural presenta la misma tendencia, debido a que después se conservaron varias de las vibraciones características de los grupos funcionales principales presentes en la cadena.

Teniendo en cuanta la Tabla 4, la relación entre el tiempo y el porcentaje de remoción es inversamente proporcional, esto puede ser ocasionado porque el fluido con un contenido constante de contaminante, que es alimentado a la columna, es inicialmente removido en la zona inferior del lecho, por lo que la solución a la salida no contiene adsorbato en este punto. Sin embargo, a medida que aumenta el tiempo de residencia el ion se va ligando al adsorbente en zonas cada vez más altas, lo cual se traduce en el movimiento de la zona de transferencia de masa con el tiempo; por consiguiente, una vez la zona de transferencia de masa se ubica en la parte más alta de la columna, quiere decir que se ha saturado el lecho [10, 15, 19]. 
Estudiando el comportamiento dinámico de la columna de lecho fijo, se obtuvieron $t_{\text {rup }}$ de 0 y 120 min para la altura del lecho de $4 \mathrm{~cm}$ y $7.5 \mathrm{~cm}$ un tiempo de ruptura de 0 y 120 min, respectivamente, como se muestra en la Tabla 5. Por lo tanto, el aumento de la altura del lecho favorece el tiempo de ruptura, ya que aumenta la cantidad de sitios de transferencia de masa. Además, se estableció que el cambio del $t_{\text {rup }}$ es proporcional a la altura del lecho, lo cual significa que se adsorbe mayor cantidad de soluto por gramo de adsorbente, lo que es coherente con los porcentajes de remoción presentados. Con base en este desempeño, es posible afirmar que el proceso de adsorción de iones de $\mathrm{Hg}$ (II), sobre un lecho de cacao, es dependiente de la altura, ya que el incremento en la altura del lecho también significa un aumento el área superficial disponible y el tiempo de contacto entre los iones de $\mathrm{Hg}$ y el sorbente $[13,15]$.

$\mathrm{Al}$ modelar los datos experimentales de las curvas de ruptura se obtuvo que el modelo de Thomas es el que mejor los ajusta; estos resultados son coherentes con resultados obtenidos por otros investigadores en estudios similares; este ajuste es apropiado para flujo constante en columna sin dispersión axial, y su comportamiento coincide con el modelo de isoterma de Langmuir (adsorción en monocapa) y modelo cinético reversible de pseudo-segundo orden; además, se observa una relación proporcional entre la altura del lecho y la constante de velocidad de Thomas ( $\left.k_{\mathrm{TH}}\right)$, lo cual describe un aumento en velocidad de transporte del ion de la solución acuosa al adsorbente y un aumento de $\mathrm{k}_{\mathrm{TH}}$ y $\mathrm{q}_{\mathrm{e}}$, como se muestra en la Tabla 6 [15-18].

\section{CONCLUSIONES}

Teniendo en cuenta el análisis FTIR se estableció que, el grupo funcional característico en la adsorción de iones metálicos presentes en solución acuosa es el grupo hidroxilo (OH-), el cual se encuentra en la superficie de la cáscara de cacao. Los ensayos de adsorción evidenciaron que la biomasa residual de cacao es eficiente como adsorbente de $\mathrm{Hg}$ (II), removiéndose hasta el $99.62 \%$ del contaminante usándose una columna con un lecho de $7.5 \mathrm{~cm}$. Se determinó que la altura del lecho es una variable que influye de manera proporcional con el tiempo de servicio, siendo el modelo de Thomas el que mejor ajustó los datos experimentales de adsorción.

\section{AGRADECIMIENTOS}

A la Universidad de Cartagena por facilitar la ejecución del proyecto que dio origen a este artículo; tesis de pregrado titulada "Adsorción de plomo, mercurio, níquel y cadmio en columnas de lecho fijo sobre cáscaras de cacao", en el programa de Ingeniería Química.

\section{REFERENCIAS}

[1] M. F. Castillo, M. Ramírez, R. S. García, M. Bernal, B. Espinosa, J. A. Solís, C. Durán,"Reaprovechamiento integral de residuos agroindustriales: cáscara y pulpa de cacao para la producción de pectinas," Rev. Latinoam. el Ambient. y las Ciencias, vol. 1, no. 2, pp. 45-66, 2010.

[2] J. G. Ramírez Gil, "Pérdidas económicas asociadas a la pudrición de la mazorca del cacao causada por Phytophthora spp., y Moniliophthora roreri (Cif y Par) Evans et al., en la hacienda Theobroma, Colombia," Rev. Protección Veg., vol. 31, no. 1, pp. 42-49, 2016.

[3] N. J. Cárdenas Pardo, A. E. Darghan Contreras, M. D. Sosa Rico, and A. Rodríguez, "Análisis espacial de la incidencia de enfermedades en diferentes genotipos de cacao (Theobroma cacao L.) en El Yopal (Casanare), Colombia," Acta Biológica Colomb., vol. 22, no. 2, pp. 209-220, 2017.

[4] C. Tejada, A. Herrera, and E. Ruiz, "Kinetic and isotherms of biosorption of $\mathrm{Hg}$ ( II ) using citric acid treated residual materials Cinética e isotermas de bioadsorción de $\mathrm{Hg}$ ( II ) usando materiales residuales tratados con ácido cítrico," Ing. y Competividad, vol. 18, no. 1, pp. 117-127, 2016. 
[5] C. Tejada, Á. Villabona, and J. Núñez, "Uso de biomasas para la adsorción de plomo , níquel , mercurio y cromo," Univ. Cart. Colomb., vol. 9, pp. 41-51, 2015.

[6] C. Tejada-Tovar, Á. Villabona-Ortiz, and L. Garcés-Jaraba, "Adsorción de metales pesados en aguas residuales usando materiales de origen biológico," Tecno Lógicas, vol. 18, no. 34, pp. 109-123, 2015.

[7] V. R. García, N. Borja, E. Guzmán, A. G. Yipmantin, and H. Maldonado, "Equilibrio de biosorción de plomo (II) y caracterización mediante FT-IR y SEM-EDAX en pectina reticulada proveniente de cáscaras de naranj," Rev. Soc. Química Peru, vol. 79, no. 3, pp. 256-264, 2013.

[8] C. Tejada Tovar, Á. Villabona Ortiz, and V. Ruiz Rangel, "Biomasa residual para remoción de mercurio y cadmio: una revisión," Ingenium, vol. 6, no. 14, pp. 11-21, 2012.

[9] C. T. Tovar, Á. V. Ortiz, and M. J. Villadiego, "Remoción de cromo hexavalente sobre residuos de cacao pretratados químicamente," Rev. U.D.C.A Act. Div. Cient., vol. 20, no. 1, pp. 139-147, 2017.

[10] S. Mädler, F. Sun, C. Tat, N. Sudakova, P. Drouin, R. J. Tooley, E. J. Reiner, T. A. Switzer, R. Dyer, H. M. Skip Kingston, M. Pamuku and V. I. Furdui "Trace-Level Analysis of Hexavalent Chromium in Lake Sediment Samples Using Ion Chromatography Tandem Mass Spectrometry," J. Environ. Prot. (Irvine,. Calif)., vol. 7, no. February, pp. 422-434, 2016.

[11] X. Li, H. Zhou, W. Wu, S. Wei, Y. Xu, and Y. Kuang, "Studies of heavy metal ion adsorption on Chitosan/Sulfydryl-functionalized graphene oxide composites," J. Colloid Interface Sci., vol. 448, pp. 389-397, 2015.

[12] R. Kumar, D. K. Arya, N. Singh, and H. K. Vats, "Removal of Cr (VI) Using Low Cost Activated Carbon Developed By Agricultural Waste," IOSR J. Appl. Chem., vol. 10, no. 1, pp. 76-79, 2017.
[13] S. Rangabhashiyam and N. Selvaraju, "Adsorptive remediation of hexavalent chromium from synthetic wastewater by a natural and $\mathrm{ZnCl} 2$ activated Sterculia guttata shell," $J$. Mol. Liq., vol. 207, pp. 39-49, 2015.

[14] M. Hadavifar, N. Bahramifar, H. Younesi, and Q. Li, "Adsorption of mercury ions from synthetic and real wastewater aqueous solution by functionalized multi-walled carbon nanotube with both amino and thiolated groups," Chem. Eng. J., vol. 237, pp. 217-228, 2014.

[15] J. Lara, C. Tejada, A. Villabona, and A. Arrieta, "Adsorción de plomo y cadmio en sistema continuo de lecho fijo sobre residuos de cacao," Rev. Ion, vol. 29, no. 2, pp. 111-122, 2016.

[16] C. Tejada Tovar, A. Villabona Ortiz, and E. Ruiz Paternina, "Cinética de adsorción de $\mathrm{Cr}$ (VI) usando biomasas residuales modificadas químicamente en sistemas por lotes y continuo," Rev. Ion, vol. 28, no. 1, pp. 29-41, 2015.

[17] S. M. El-Bahy and Z. M. El-Bahy, "Immobilization of 2-amino pyridine onto poly(acrylonitrile-co-N, $\mathrm{N}^{\prime}$ -

methylenebisacrylamide) nanoparticles for the removal of $\mathrm{Hg}$ (II), $\mathrm{Cd}(\mathrm{II})$ and $\mathrm{Cr}(\mathrm{III})$ : Batch and column techniques," J. Environ. Chem. Eng., vol. 5, no. 4, pp. 3560-3571, 2017.

[18] J. L. Gong, L. Zhang, Y. Jiang, G. M. Zeng, Z. H. Cui, K. Liu, C. H. Deng, Q. Y. Niu, J. H. Deng and S. Y. Huan, "Continuous adsorption of $\mathrm{Pb}(\mathrm{II})$ and methylene blue by engineered graphite oxide coated sand in fixed-bed column," Appl. Surf. Sci., vol. 330, pp. 148-157, 2015.

[19] O. Allahdin, J. Mabingui, M. Wartel, and A. Boughriet, "Removal of $\mathrm{Pb} 2+$ ions from aqueous solutions by fixed-BED column using a modified brick: (Micro)structural, electrokinetic and mechanistic aspects," Appl. Clay Sci., vol. 148, no. July, pp. 56-67, 2017. 